\title{
Editorial
}

\section{Cardiac Electromechanical Abnormalities in Hemodialysis Patients: Indicators of Cardiomyopathy and Future Risk?}

\author{
Mohammad Kazem Fallahzadeh $^{\mathrm{a}}$ Peter A. McCullough ${ }^{\mathrm{a}-\mathrm{d}}$ \\ a Baylor University Medical Center, ${ }^{b}$ Baylor Heart and Vascular Institute, and ${ }^{\mathrm{c} B a y l o r ~ J a c k ~ a n d ~ J a n e ~ H a m i l t o n ~ H e a r t ~}$ \\ and Vascular Hospital, Dallas, Tex., and ${ }^{\mathrm{d}}$ The Heart Hospital Baylor Plano, Plano, Tex., USA
}

Cardiovascular diseases (CVDs) are more common in chronic kidney disease and end-stage renal disease (ESRD) patients as compared with general population and are a major of cause of morbidity and mortality in this population $[1,2]$. CVD accounts for approximately $50 \%$ of mortality in ESRD patients. In addition to the increased chance of coronary artery disease, the chance of developing arrhythmic events and sudden cardiac death is also higher in ESRD patients $[3,4]$. The underlying pathophysiology behind this increased chance of arrhythmic event in hemodialysis patients is not clearly understood. Previous studies have shown that hemodialysis patients have lower left ventricular ejection fraction and higher atrial diameters as compared with general population [57]. Using the timing of electrocardiogram and tissue Doppler on echocardiography, the time from electrical activation to muscular contraction can be assessed. Atrial electromechanical delay (AEMD) times have been reported to be longer in ESRD patients and have been shown to improve after each hemodialysis session [6-8]. Additionally, prolonged atrial conduction times are considered as predisposing factors for atrial fibrillation [9]. In this issue of Journal, Turkmen et al. [10] from Turkey have compared the cardiac electromechanical characteristics and biochemical profile of 60 hemodialysis patients versus 44 healthy controls. They also followed the hemo- dialysis patients for 2 years and compared the characteristics of 19 patients who died within this 2 -year period versus 41 living hemodialysis patients.

Consistent with previous reports, this study showed that hemodialysis patients have significantly longer AEMD times as compared with healthy controls. However, the novel finding of this study was that deceased hemodialysis patients were found to have longer left intra-atrial conduction delay (LIAT) and interatrial EMD times as compared with living hemodialysis patients. Moreover, increased LIAT, interatrial time and C-reactive protein levels (as a marker of inflammation) and decreased albumin levels (as a marker of malnutrition) were found to be associated with increased risk of combined cardiovascular events. The observed increased AEMD times in deceased hemodialysis patients and the positive association of increased LIAT and interatrial time with cardiovascular outcomes could provide a novel explanation for the propensity of arrhythmic events in hemodialysis patients. The prolonged AEMD times in these patients could possibly be due to uremic dysfunction of cardiomyocytes, accelerated apoptosis and autophagy and replacement interstitial matrix/fibrosis that results in slowed electromechanical conduction due to the decreased density of contractile fibers. Inflammation and malnutrition in hemodialysis patients could possibly

\section{KARGER 125}

(c) 2015 S. Karger AG, Base

0250-8095/15/0423-0237\$39.50/0 
contribute to this process and potentially be amenable to therapy.

This study has several limitations, and therefore, its results should be interpreted cautiously. The most important limitations of this study are its small sample size and short duration of follow-up. The confounding effect of the medical therapy was also not considered in evaluation of association of AEMD times and cardiovascular outcomes. Moreover, due to sparse data, the authors did not evaluate the association of patients' characteristic with each cardiovascular outcome separately. Instead, they used a combined cardiovascular outcome measure comprising of all cardiovascular deaths, myocardial infarctions and atrial fibrillation events.
Future studies with larger sample sizes and longer durations of follow-up are needed to evaluate the association of AEMD times with different cardiovascular outcomes such as death due to CVDs, myocardial infarction, sudden cardiac death, atrial fibrillation and other arrhythmic events. Moreover, future studies should attempt to uncover the degree of reversibility of this measure to better understand the dynamic pathobiologic nature of cardiac dysfunction in patients with ESRD.

\section{Disclosure Statement}

The authors have no conflicts of interest.

\section{References}

1 Herzog CA, Asinger RW, Berger AK, et al: Cardiovascular disease in chronic kidney disease. A clinical update from kidney disease: improving global outcomes (KDIGO). Kidney Int 2011;80:572-586.

2 McCullough PA: Cardiovascular disease in chronic kidney disease from a cardiologist's perspective. Curr Opin Nephrol Hypertens 2004;13:591-600.

3 Saravanan P, Davidson NC: Risk assessment for sudden cardiac death in dialysis patients. Circ Arrhythm Electrophysiol 2010;3:553559.
4 Genovesi S, Valsecchi MG, Rossi E, et al: Sudden death and associated factors in a historical cohort of chronic haemodialysis patients. Nephrol Dial Transplant 2009;24:2529-2536.

5 Zoccali C, Benedetto FA, Mallamaci F, et al: Prognostic value of echocardiographic indicators of left ventricular systolic function in asymptomatic dialysis patients. J Am Soc Nephrol 2004; 15:1029-1037.

6 Tekce H, Ozturk S, Aktas G, et al: The effects of a single dialysis session on atrial electromechanical conduction times and functions. Kidney Blood Press Res 2013;37:622-630.

7 Tekce H, Ozturk S, Aktas G, et al: Atrial electrical and mechanical dysfunction in non-diabetic, non-hypertensive hemodialysis patients. Acta Medica 2014;30:21.
8 Karavelioğlu Y, Karapınar H, Özkurt S, et al: Evaluation of atrial electromechanical coupling times in hemodialysis patients. Echocardiography 2014;31:449-455.

9 Todaro MC, Choudhuri I, Belohlavek M, et al: New echocardiographic techniques for evaluation of left atrial mechanics. Eur Heart J Cardiovasc Imaging 2012;13:973-984.

10 Turkmen K, Demirtas L, Topal E, Gaipov A, Kocyigit I, Orscelik O, Guney I, Kilic S, Yilmiz MI: Predictive value of atrial electromechanical delay times for 2 year combined cardiovascular events in hemodialysis patients. Am J Nephrol 2015;42:239-249. 